\title{
Synthesis and characterization of novel push-pull thiophene and thienylpyrrole derivatives functionalized with indanonedicyanovinyl acceptor moiety as efficient NLO-chromophores
}

\author{
Rosa M. F. Batista , Susana P. G. Costa , Michael Belsley ${ }^{1}$, M. Manuela M. Raposo ${ }^{* 1}$ \\ ${ }^{1}$ Centre of Chemistry, University of Minho, Campus of Gualtar, 4710-057 Braga, Portugal \\ ${ }^{2}$ Centre of Physics, University of Minho, Campus of Gualtar, 4710-057 Braga, Portugal
}

\begin{abstract}
The synthesis and characterization of new chromophores with second-order nonlinearities containing thienylpyrrole 1a, 2a-b, bithiophene $\mathbf{3}$ and arylthiophene $\mathbf{4}$ as $\pi$-conjugated bridges and indanonedicyanovinyl acceptor group are reported. The effect of placing the acceptor group at thiophene or pyrrole rings on the optoelectronic properties was also evaluated for thienylpyrrole derivatives 1a and 2a-b. The linear optical properties (absorption and emission) for all compounds were evaluated in dioxane solutions. In addition, the hyperpolarizabilities $\beta$ of chromophores 1-4 were measured using hyper-Rayleigh scattering in dioxane solutions and thermogravimetric analysis (TGA) was used to evaluate their thermal stability. The experimental results indicate that chromophores 1-4 are endowed with both excellent optical nonlinearities and high thermal stability making them interesting candidates for nonlinear optical (NLO) applications.
\end{abstract}

Keywords: nonlinear optics (NLO), hyper-Rayleigh scattering (HRS) technique, bithiophene, arylthiophene, thienylpyrrole, indanonedicyanovinyl, donor-acceptor pi-conjugated chromophores, thermal stability.

\section{INTRODUCTION}

An exciting aspect of nanoscience is that relationships between structure and electronic properties are being uncovered through a combination of synthesis, optical and structural characterization and theory. This is especially true regarding the design of organic molecules as nonlinear optical (NLO) materials. Motivated by the great potential of organic molecules for use in optical communication, information processing, frequency doubling and integrated optics, there has been intense efforts in recent years to optimize the nonlinear response of donor-acceptor heterocyclic systems. These systems are especially interesting because they possess an enhanced NLO response in comparison with the corresponding aryl analogues. Electron excessive/deficient heterocycles can act as auxiliary donors/acceptors when they are connected to donating/withdrawing groups, and the increase of donor/acceptor strength can lead to a substantial increase in the NLO response. ${ }^{2-3}$ We have recently synthesized and characterized several bithiophene and thienylpyrrole push-pull heterocyclic compounds for several optical applications. ${ }^{4-9}$ Here we report on the synthesis, thermal stability and linear and NLO properties characterization of novel thienylpyrroles 1-2, bithiophene $\mathbf{3}$ and arylthiophene $\mathbf{4}$ functionalized with indanonedicyanovinyl acceptor group at thiophene or pyrrole 
rings. The molecular structure, especially the degree of planarity and the relative orientations of the auxiliary donor and acceptor entities, is found to strongly influence the NLO response.

\section{EXPERIMENTAL DETAILS}

\subsection{Measurements}

Hyper-Rayleigh scattering (HRS $)^{10-11}$ was used to characterize the first hyperpolarizability $\beta$ of response of the molecules studied. The experimental set-up is schematically illustrated in Fig. 1. A Q-switched Nd:YAG laser operating at the fundamental wavelength of $1064 \mathrm{~nm}$ was used to create hyper-Rayleigh scattered light at $532 \mathrm{~nm}$ from the samples. The pulses had energies of approximately $10 \mathrm{~mJ}$ and a duration (FWHM) close to $12 \mathrm{~ns}$ and was vertically polarized. The incident power could be varied using a combination of a half wave-plate and Glan polarizer. The incident beam was weakly focused (beam diameter $\sim 0.5 \mathrm{~mm}$ ) into the solution contained in a $5 \mathrm{~cm}$ long cuvette. The hyper- Rayleigh signal was collected at right angles to the incident beam and collimated using a high numerical aperture lens (N.A. $=0.8$ ) without polarization selection. Subsequently the signal was spectrally discriminated using a combination of an infrared blocking filter and one of two narrow band interference filters before being detected by a photomultiplier (Hamamatsu model H9305-04). The current pulse from the photomultiplier was integrated using a Stanford Research Systems gated box-car integrator (model SR250) and normalized pulse by pulse to the second harmonic signal from a $1 \mathrm{~mm}$ quartz plate in order to compensate for fluctuations in the temporal profile of the laser pulses due to longitudinal mode beating. Dioxane was used as a solvent, and the $\beta$ values were calibrated using a reference solution of $p$-nitroaniline $(p \mathrm{NA})^{12-13}$ also dissolved in dioxane at a concentration of $1 \times 10^{-2} \mathrm{~mol} \mathrm{dm}^{-3}$ (external reference method). The concentrations of the solutions under study were chosen so that the corresponding hyper-Rayleigh signals fell well within the dynamic range of the detection system. All solutions were filtered $(0.2$ $\mu \mathrm{m}$ porosity) to avoid spurious signals from suspended impurities.

Other characterization measurements were carried out as follows. IR spectra were determined on a BOMEM MB 104 spectrophotometer using KBr discs. UV-visible absorption spectra (200 - 700 $\mathrm{nm}$ ) were obtained using a Shimadzu UV/2501PC spectrophotometer and the fluorescence spectra were collected using a FluoroMax-4 spectrofluorometer. Thermogravimetric analysis of samples was carried out using a TGA instrument model Q500 from TA Instruments, under high purity nitrogen supplied at a constant $50 \mathrm{~mL} \mathrm{~min}^{-1}$ flow rate. All samples were subjected to a $20{ }^{\circ} \mathrm{C} \mathrm{min}$ meating $^{-1}$ he rate and were characterized between 25 and $500{ }^{\circ} \mathrm{C}$.

\subsection{Calculations}

The hyperpolarizability of $p$ NA dissolved in dioxane is known from EFISH measurements carried out at the same fundamental wavelength. The small hyper-Rayleigh signal that arises from dioxane was taken into account according to the equation (1)

$$
I_{2 \omega}=G\left(N_{\text {solvent }}\left\langle\beta_{\text {solvent }}^{2}\right\rangle+N_{\text {solute }}\left\langle\beta_{\text {solute }}^{2}\right\rangle\right) I_{\omega}^{2}
$$


where the factor $G$ is an instrumental factor that takes into account the detection efficiency (including geometrical factors and linear absorption or scattering of the second harmonic light on its way to the detector) as well as local field corrections due to the solvent. We took particular care to avoid reporting artificially high hyperpolarizibilities due to a possible contamination of the hyper Rayleigh signal by molecular fluorescence near $532 \mathrm{~nm}$. Measurements were carried out using two different interference filters with different transmission pass bands centred near the second harmonic at $532 \mathrm{~nm}$. By comparing the signals obtained with the two different filters we can determine the relative contributions of the hyper-Rayleigh and fluorescence signals if present. Details concerning this procedure can be found elsewhere.

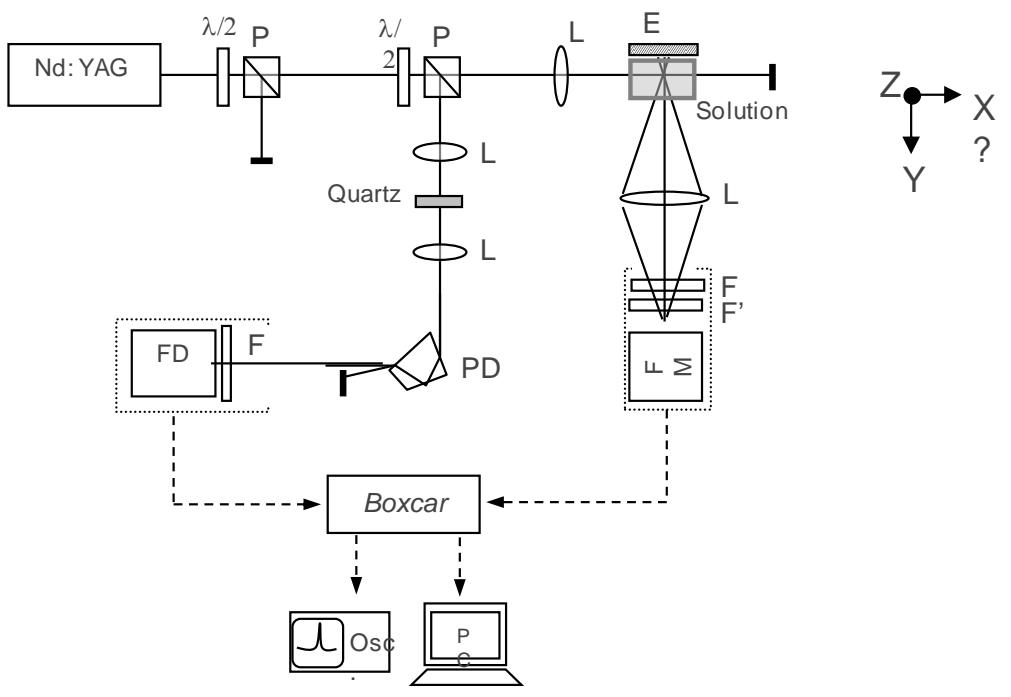

Fig. 1. Scheme of assembly for measurements of diffusion hyper-Rayleigh. $\mathrm{P}=$ polarizer, $\lambda / 2=$ half-waveplate, $\mathrm{L}=$ lens, $\mathrm{E}=$ mirror, $\mathrm{PD}=$ disperser prism, $\mathrm{F}^{\prime}=$ band-pass filter, $\mathrm{F}=$ low-pass filter, $\mathrm{FD}=$ photodetector, $\mathrm{FM}=$ photomultiplier.

After subtracting off the small second harmonic signal due to the solvent and carrying out the decomposition of the remaining signal into second harmonic and fluorescent components we estimate the first hyperpolarizability of the molecules under study using equation (2)

$$
\beta=\beta_{p N a} \sqrt{\frac{S_{c h r o m}^{h r s}}{S_{p N a}^{h r s}} \frac{N_{p N a}}{N_{c h r o m}}} .
$$

Here $S_{\text {chrom }}^{\text {hrs }}$ is the normalized hyper-Rayleigh component of the signal from the chromophore while $S_{p N a}^{h r s}$ is the signal from the $p$-nitroaniline $(p \mathrm{NA})$ solution used as an external reference acquired under identical experimental conditions. The molar densities of the respective solutions are $N_{\text {chrom }}$ and $N_{p N a}$. In using this expression we assume, as is typical, that the hyperpolarizability tensor is 
dominated by a single longitudinal element. This is the case for the reference molecule, $p$ NA with a value of $\beta_{z z z}=16.9 \times 10^{-30}$ esu as measured using the EFISH technique. ${ }^{12,13}$

\subsection{Synthesis}

\section{RESULTS}

The new push-pull heterocyclic systems 1-4 (Fig. 2) bearing different $\pi$-conjugated bridges (thienylpyrrole, bithiophene and arylthiophene) functionalized with the same 2-(3-oxo-2,3dihydroinden-1-ylidene)malononitrile acceptor moiety at pyrrole or thiophene heterocycles were synthesised in order to evaluate the effect of the electronic nature of the $\pi$-conjugated bridge and also the position of substitution of the acceptor group at the heterocyclic system on the nonlinear optical properties of chromophores 1-4. These compounds were obtained in good to excellent yields (72$96 \%$ ) by condensation of the formyl- precursors: bithiophenes, ${ }^{14}$ thienylpyrroles ${ }^{15}$ and arylthiophenes, ${ }^{16}$ with 3-oxo-2,3-dihydroinden-1-ylidene)malononitrile in ethanol at room temperature for 5 days (Table 1). The precipitate obtained was recrystallized from ethanol. The structures of the new derivatives 1-4 were confirmed by the usual spectroscopic techniques. The details of the synthesis and full characterization of compounds 1-4 will be reported elsewhere.

Table 1. Yields, IR spectrocospic data and $\mathrm{T}_{d}$ data for chromophores 1-4.

\begin{tabular}{|ccccc|}
\hline & Yield & \multicolumn{2}{c|}{$v^{\mathrm{a}}\left(\mathrm{cm}^{-1}\right)$} & $\mathrm{T}_{d}$ \\
Compound & $(\%)$ & $\mathrm{C}=\mathrm{O}$ & $\mathrm{C} \equiv \mathrm{N}$ & $\left({ }^{\circ} \mathrm{C}\right)^{\mathrm{c}}$ \\
\hline $\mathbf{1 a}$ & 72 & 1693 & 2209 & 298 \\
$\mathbf{2 a}$ & 86 & 1698 & 2213 & 215 \\
$\mathbf{2 b}$ & 89 & $1699^{\mathrm{b}}$ & $2217^{\mathrm{b}}$ & 252 \\
$\mathbf{3}$ & 72 & 1695 & 2214 & 306 \\
$\mathbf{4}$ & 96 & 1699 & 2215 & 339 \\
\hline
\end{tabular}

${ }^{\text {a }}$ The IR spectra were obtained in $\mathrm{KBr}$.

${ }^{\mathrm{b}}$ The IR spectra were obtained in Nujol.

${ }^{\mathrm{c}}$ Decomposition temperature $\left(\mathrm{T}_{d}\right)$ measured at a heating rate of $20{ }^{\circ} \mathrm{C} \mathrm{min}^{-1}$ under a nitrogen atmosphere, obtained by thermogravimetric analysis (TGA).
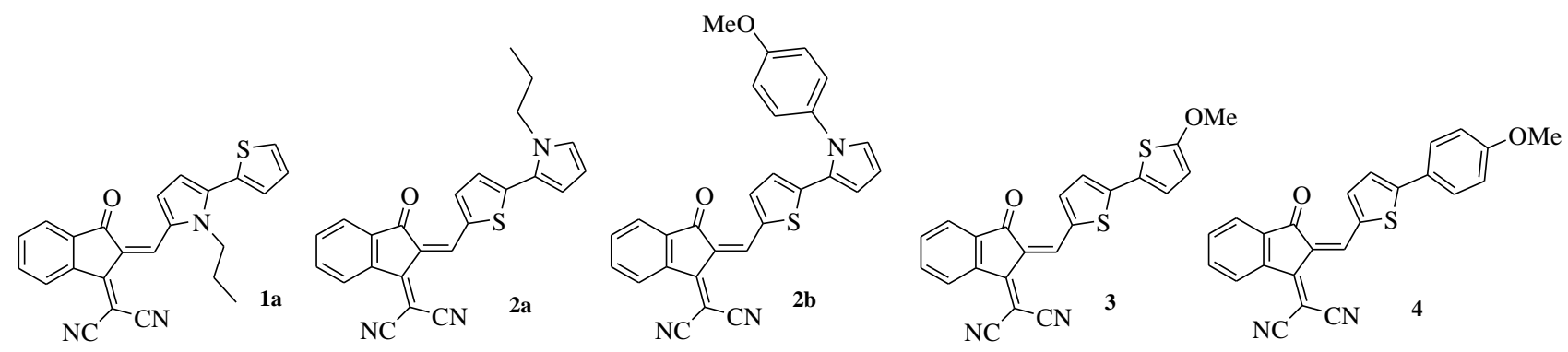

Fig. 2. Chemical structures of the chromophores 1-4. 


\subsection{UV-visible study}

Electronic absorption spectra of chromophores 1-4 in dioxane solutions $\left(10^{-4} \mathrm{M}\right)$ showed an intense lowest energy charge-transfer absorption band in the UV-visible region in the range 521-573 $\mathrm{nm}$ (Table 2). The position of this band was strongly influenced by the position of attachment of the indanonedicyanovinyl unit, being $c a .30 \mathrm{~nm}$ red-shifted for the attachment at the thiophene ring (2a, $\left.\lambda_{\max }=573 \mathrm{~nm}\right)$ when compared to the attachment at the pyrrole ring $\left(1 \mathrm{a}, \lambda_{\max }=546 \mathrm{~nm}\right)($ Fig. 3 , left). The electronic character of the substituent at the pyrrole nitrogen (propyl or 4-methoxyphenyl group) did not have a marked influence as compounds $\mathbf{2 a}$ and $\mathbf{2 b}$ showed similar maximum wavelength of absorption (compare entries 2 and 3 in Table 2). As previously stated, electron excessive/deficient heterocycles can act as auxiliary donors/acceptors when they are connected to donating/withdrawing groups, increasing the overall donor/acceptor strength of the system, and this is confirmed by comparing the effect on $\lambda_{\max }$ of the replacement of a thiophene ring $\left(3, \lambda_{\max }=564\right.$ $\mathrm{nm})$ by a phenyl ring $\left(4, \lambda_{\max }=521 \mathrm{~nm}\right)$ (Fig. 3, right).
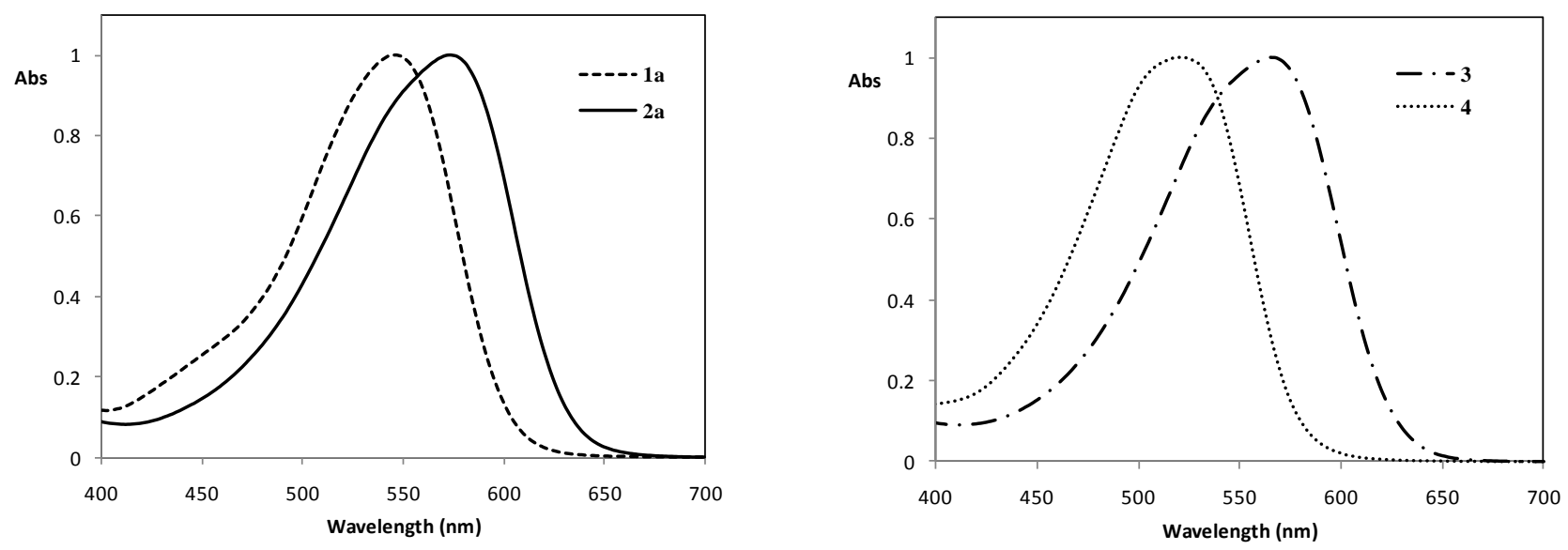

Fig. 3. UV-visible absorption normalized spectra of compounds $\mathbf{1 a}$ and $\mathbf{2 a}$ (left) and compounds $\mathbf{3}$ and $\mathbf{4}$ (right) in dioxane.

This effect was also seen in the comparison of $\mathbf{2 b}$ and $\mathbf{4}$ (entries 3 and 5 in Table 2), in which the conjugation pathway was further extended by incorporation of a pyrrole ring to the $\pi$-conjugated system $\left(2 \mathrm{~b}, \lambda_{\max }=567 \mathrm{~nm}\right)$ resulting in a red-shift when compared to compound $4\left(\lambda_{\max }=521 \mathrm{~nm}\right)$, potentiating the inductive and conjugative effect of the methoxy group. As is typical in charge transfer molecules, an increase in the wavelength of the absorption maximum $\lambda_{\max }$ in the UV-visible spectra is accompanied by an increase in the first hyperpolarizability $\beta$ values, characteristic of the strength of the second order NLO response. ${ }^{1}$ The same trends were seen in the fluorescence data of chromophores 1-4 in dioxane, with the wavelength of maximum emission appearing between 583$640 \mathrm{~nm}$. 


\subsection{Nonlinear optical study}

We used the hyper-Rayleigh scattering method to determine the first hyperpolarizabilities, $\beta$, of chromophores 1-4 in dioxane solutions (Table 2). $p$-Nitroaniline ( $p$ NA) was used as standard ${ }^{12-13}$ in order to obtain quantitative values while care was taken to properly account for possible fluorescence of the dyes (see the experimental details). To take into account possible resonant enhancement due to the proximity of the incident or second-harmonic light to the energy of the charge transfer transition of the molecule, one usually extrapolates the measured hyperpolarizabilities to the dc (static) limit, $\beta_{0}$, using the two-level model of Oudar and Chelma (equatin 3), ${ }^{22}$

$$
\beta_{0}=\beta\left[1-\left(\lambda_{\max } / 1064 n m\right)^{2}\right]\left[1-\left(\lambda_{\max } / 532 n m\right)^{2}\right]
$$

where $\lambda_{\max }$ is the peak wavelength of the UV-vis absorption profile. These static hyperpolarizabilities neglect damping or any influence of higher excited states. They are therefore only indicative and should be treated with caution.

Table 2. UV-vis absorption and emission data, $\beta$ and $\beta_{0}$ values data for chromophores 1-4 in dioxane.

\begin{tabular}{|ccccccc|}
\hline Entry & Compound & $\lambda_{\max }(\mathrm{nm})$ & $\log \varepsilon$ & $\lambda_{\text {emi }}(\mathrm{nm})$ & $\begin{array}{c}\beta \\
\left(10^{-30} \mathrm{esu}\right)^{\mathrm{a}}\end{array}$ & $\begin{array}{c}\beta_{0} \\
\left(10^{-30} \mathrm{esu}\right)^{\mathrm{b}}\end{array}$ \\
\hline 1 & $\mathbf{1 a}$ & 546 & 4.40 & 605 & 350 & $14 \pm 2$ \\
2 & $\mathbf{2 a}$ & 573 & 4.62 & 640 & 905 & $103 \pm 7$ \\
3 & $\mathbf{2 b}$ & 567 & 4.58 & 631 & 810 & $79 \pm 8$ \\
4 & $\mathbf{3}$ & 564 & 4.61 & 633 & 1125 & $100 \pm 6$ \\
5 & $\mathbf{4}$ & 521 & 4.59 & 583 & 1100 & $34 \pm 3$ \\
6 & $p \mathrm{NA}$ & 352 & --- & --- & $16.9^{12-13}$ & 8.5 \\
\hline
\end{tabular}

a All compounds are transparent at the $1064 \mathrm{~nm}$ fundamental wavelength.

${ }^{\mathrm{b}}$ Data corrected for resonance enhancement at $532 \mathrm{~nm}$ using the two-level model with $\beta_{0}=\beta\left[1-\left(\lambda_{\max } / 1064\right)^{2}\right]\left[1-\left(\lambda_{\max } / 532\right)^{2}\right]$; damping factors not included $1064 \mathrm{~nm}^{21-23}$

Comparison of the $\beta$ values for $2 \mathbf{a}\left(905 \times 10^{-30} \mathrm{esu}\right)$ and $\mathbf{1 a}\left(350 \times 10^{-30} \mathrm{esu}\right)$ shows that the substitution of the 3-oxo-2,3-dihydroinden-1-ylidene)malononitrile acceptor group at the 5'-position on the thiophene ring (2a) leads to a larger nonlinearity than the same electron-deficient moiety at 5position on the pyrrole ring (1a). The results obtained show that the location of the electron acceptor group on the pyrrole or on the thiophene ring can, by itself, dramatically alter the overall molecular nonlinearity of the system. One must therefore view the thienylpyrrole system not simply as the conjugated bridge but also as one of the key structural units, affecting the overall electron transfer properties of the system. Pyrrole, being the most electron-rich of the five-membered heterocyclic rings, counteracts the electron withdrawing effect of the acceptor group (in 1a) leading to a decrease 
in $\beta$. These findings are consistent with previous theoretical ${ }^{17-18}$ and experimental ${ }^{19}$ studies, $^{2}$ including our recent work. ${ }^{4-6,9}$ In general, it can be concluded that, depending on the location of the acceptor groups in the system, an increase or decrease in the molecular nonlinear activity of thienylpyrrole push-pull chromophores can be obtained. By comparing the $\beta$ values for the molecules $\mathbf{2 a}\left(905 \times 10^{-30} \mathrm{esu}\right)$ and $\mathbf{2 b}\left(810 \times 10^{-30} \mathrm{esu}\right)$, it can be seen that the substitution of a 4methoxyphenyl group by the $n$-propyl group at position 1 of pyrrole ring leads to a higher first hyperpolarizability, which is probably due to the greater planarity of compound $\mathbf{2} \mathbf{a}^{9,20}$ facilitating the charge transfer upon excitation. Compounds 3 and $\mathbf{4}$ bearing bithiophene $\left(1125 \times 10^{-30}\right.$ esu $)$ and arylthiophene $\left(1100 \times 10^{-30} \mathrm{esu}\right)$ conjugated bridges display still greater $\beta$ values when compared to thienylpyrrole derivatives. These values are exceptionally large. Even the extrapolated response $\beta_{0}$ of chromophore $\mathbf{3}$ is more than an order of magnitude higher than that of the standard reference molecule $p$-nitroaniline.

\subsection{Thermal properties}

The thermal stability of chromophores 1-4 was estimated by thermogravimetric analysis (Table 1). All samples had high decomposition temperatures $\left(\mathrm{T}_{d}=215-339{ }^{\circ} \mathrm{C}\right)$, measured at heating rate of 20 ${ }^{\circ} \mathrm{C} \min ^{-1}$. Thienylpyrrole 1a bearing a pyrrole ring substituted at positions 2 and 5, bithiophene 3 and arylthiophene $\mathbf{4}$ are the more thermally stable compounds exhibiting higher decomposition temperatures $\left(\mathrm{T}_{d}=298-339^{\circ} \mathrm{C}\right)$. This thermal stability would benefit the practical applications of these chromophores on optical devices.

\section{CONCLUSIONS}

In summary, we have synthesized new push-pull heterocyclic systems 1-4 in good to excellent yields. Hyper-Rayleigh scattering was used to determine the first hyperpolarisability, $\beta$, the data showing that $\beta$ is dependent on the substituent on the pyrrole ring (alkyl or aryl) and on the position of substitution of the acceptor group at the pyrrole or at the thiophene ring. It was also shown that the thienylpyrrole derivatives functionalized with the acceptor group at the thiophene ring had higher molecular nonlinearities. On the other hand chromophores based on bithiophene $\mathbf{3}$ and arylthiophene 4 bridges exhibit the highest $\beta$ values and thermal stabilities. Experimental results for compounds 14, indicate that exceptional optical nonlinearities are well balanced with good thermal stability making them good candidates for NLO applications.

\section{ACKNOWLEDGEMENTS}

We are grateful to the Fundação para a Ciência e Tecnologia (Portugal) for financial support at Centre of Chemistry - University of Minho through project PTDC/QUI/66251/2006 (FCOMP-010124-FEDER-007429) and Centre of Physics - University of Minho through project PTDC/CTM/105597/2008, with funding from COMPETE/FEDER and a PhD grant to R.M.F. Batista (SFRH/BD/36396/2007). 


\section{REFERENCES}

[1] Nalwa, H. S. and Miyata, S. (Eds), [Nonlinear Optics of Organic Molecules and Polymers], CRC Press, New York, (1997).

[2] Bradamante, S., Facchetti, A. and Pagani, G. A., "Heterocycles as donor and acceptor units in push-pull conjugated molecules. Part 1", J. Phys. Org. Chem. 10, 514-524 (1997).

[3] Breitung, E. M., Shu, C.-F. and McMahon, R. J., "Thiazole and Thiophene Analogues of Donor-Acceptor Stilbenes: Molecular Hyperpolarizabilities and Structure-Property Relationships”, J Am Chem Soc 122, 1154-1160 (2000).

[4] Raposo, M. M. M., Sousa, A. M. R. C., Kirsch, G., Cardoso, P., Belsley, M., de Matos Gomes, E. and Fonseca, A. M. C., "Synthesis and characterization of dicyanovinyl-substituted thienylpyrroles as new NLO-chromophores", Org. Lett., 8, 3681-3684 (2006).

[5] Batista, R. M. F., Costa, S. P. G., Malheiro, E. L., Belsley, M. and Raposo, M. M. M., "Synthesis and characterization of new thienylpyrrolyl-benzothiazoles as efficient and thermally stable nonlinear optical chromophores", Tetrahedron, 63(20), 4258-4265 (2007).

[6] Batista, R. M. F., Costa, S. P. G., Belsley, M. and Raposo, M. M. M., "Synthesis and second-order nonlinear optical properties of new chromophores containing benzimidazole, thiophene and pyrrole heterocycles", Tetrahedron, 63(29), 9842-9848 (2007).

[7] Batista, R. M. F., Costa, S. P. G., Lodeiro, C., Belsley, M. and M. Raposo, M. M., "Synthesis and characterization of novel (oligo)thienyl-imidazo-phenanthrolines as versatile pi-conjugated heterocyclic systems for several optical applications nonlinear optical (NLO) chromophores", Tetrahedron, 64(39), 9230-9238 (2008).

[8] Batista, R. M. F., Costa, S. P. G., Belsley, M., Raposo, M. M. M., "Synthesis and evaluation of the optical properties of novel thermally stable phenanthrolines bearing a arylthienyl-imidazo conjugation pathway" Dyes Pigments, 80(3), 329-336 (2009).

[9] Pina, J., Melo, J. S. S., Batista, R. M. F., Costa, S. P. G. and Raposo, M. M. M., "The influence of the relative position of the thiophene and pyrrole rings in donor-acceptor thienylpyrrolyl-benzothiazole derivatives. A photophysical and theoretical investigation", Phys. Chem. Chem. Phys., 12(33), 9719-9725 (2010).

[10] Clays, K. and Persoons, A., "Hyper-Rayleigh scattering in solution” Rev. Sci. Instrum., 63(6), 3285-3289 (1992).

[11] Clays, K. and Persoons, A., "Hyper-Rayleigh scattering in solution” Phys. Rev. Lett., 66(23), 2980-2983 (1991).

[12] Teng, C. C., Garito A. F., "Dispersion of the nonlinear optical $2^{\text {nd }}$ order susceptibility of organic systems", Phys. Rev. B, 28, 6766-6773 (1983).

[13] Stahelin, M., Burland, D. M. and Rice, J. E., "Solvent dependence of the $2^{\text {nd }}$ order hyperpolarizability in paranitroaniline", Chem. Phys. Lett., 191, 245-250 (1992).

[14] Raposo, M. M. M. and Kirsch, G., "Formylation, dicyanovinylation and tricyanovinylation on 5-alkoxy- and 5amino-substituted-2,2'-bithiophenes", Tetrahedron, 59(26), 4891-4899 (2003).

[15] Raposo, M. M. M., Sousa, A.M.R.C., Fonseca, A. M. C. and Kirsch, G., "Synthesis of formyl-thienylpyrroles: versatile building blocks for NLO materials", Tetrahedron, 62(15), 3493-3501 (2006).

[16] Costa, S. P. G., Batista, R. M. F., Cardoso, P., Belsley, M. and Raposo, M. M. M.,"2-Arylthienyl-substituted 1,3benzothiazoles as new nonlinear optical chromophores", Eur. J. Org. Chem., 17, 3938-3946 (2006).

[17] Varanasi, P. R., Jen, A. K.-Y., Chandrasekhar, J., Namboothiri, I. N. N. and Rathna, A., "The Important Role of Heteroaromatics in the Design of Efficient Second-Order Nonlinear Optical Molecules: Theoretical Investigation on Push-Pull Heteroaromatic Stilbenes", J. Am. Chem. Soc., 118, 12443-12448 (1996).

[18] Ra, C. S., Kim, S. C. and Park, G., "pi-Bridging 1,3-heterocyclic aromatic rings and the first hyperpolarizability of nonlinear optical compounds", J. Mol. Struct. (Theochem), 677, 173-178 (2004).

[19] Miller, R. D., Lee, V. Y. and Moylan, C. R. "Substituted Azole Derivatives as Nonlinear Optical Chromophores", Chem. Mat., 6, 1023-1032 (1994).

[20] DeMartino, R. N., Choe, E. W., Kharanian, G., Haas, D., Leslie, T., Nelson, G., Stamatoff, J., Stuetz, D., Teng, T.T., Yoon, H. and Prasad, P.N. (Eds), [Nonlinear Optical Electroactive Polymers], Plenum Press, New York, 169-186 (1988).

[21] Oudar, J. L., "Optical nonlinearities of conjugated molecules - stilbene derivatives and highly polar aromaticcompounds", J. Chem. Phys., 67, 446-457 (1977).

[22] Oudar, J. L. and Chemla, D. S., "Hyperpolarizabilities of nitroanilines and their relations to excited-state dipolemoment", J. Chem. Phys., 66, 2664-2668 (1977).

[23] Zyss, J. and Oudar, J. L., "Relations between microscopic and macroscopic lowest-order optical nonlinearities of molecular-crystals with one-dimensional or two-dimensional units”, Phys. Rev. A, 26, 2016-2048 (1982). 